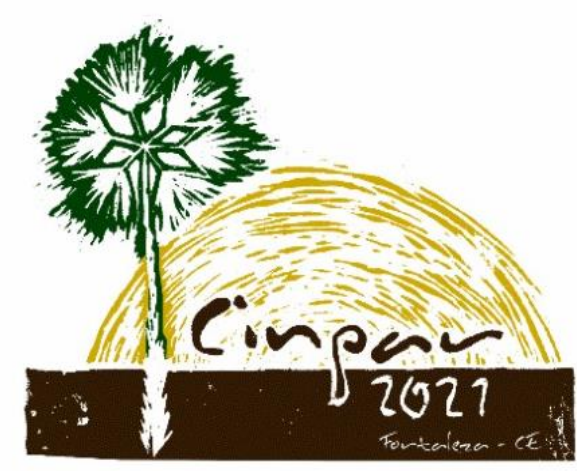

XVII Congresso Internacional sobre Patologia e Reabilitação das Construções

XVII Congreso Internacional sobre Patología y Rehabilitación de las Construcciones

XVII International Conference on Pathology and Constructions Rehabilitation

FORTALEZA (Brasil), 3 a 5 de junho de 2021 https://doi.org/10.4322/CINPAR.2021.103

\title{
Análisis comparativo entre los programas Agisoft PhotoScan y Pix4Dmapper para la identificación de fallas en pavimento flexible usando Vehículos Aéreos No Tripulados (VANTs)
}

\section{Comparative analysis between Agisoft Photoscan and Pix4Dmapper software for the identification of failures in flexible pavement using Unmanned Aerial Vehicles}

(UAVs)

Adriana Karen FERNÁNDEZ-DÁVALOS ${ }^{1}$, Camila Liliana SAAVEDRA-TÉLLEZ², José Ramiro MUÑOZ-PARDO ${ }^{3}$, Juan Pablo RAMOS-MACHUCA ${ }^{4}$, Nahúm Gamalier CAYO-CHILENO ${ }^{5}$, Joaquin Humberto Aquino ROCHA ${ }^{6}$

\footnotetext{
${ }^{1}$ Universidad Privada del Valle, Tiquipaya, Bolivia, adrita.fernan8@gmail.com

${ }^{2}$ Universidad Privada del Valle, Tiquipaya, Bolivia, camilitalilianita40@gmail.com

${ }^{3}$ Universidad Privada del Valle, Tiquipaya, Bolivia, damianft1060ft@gmail.com

${ }^{4}$ Universidad Privada del Valle, Tiquipaya, Bolivia, Pablito18ramos@gmail.com

${ }^{5}$ Universidad Privada del Valle, Tiquipaya, Bolivia, nahum.cayo.chileno@gmail.com

${ }^{6}$ Universidad Privada del Valle, Tiquipaya, Bolivia, joaquinaquinorocha@gmail.com
}

\begin{abstract}
Resumen: En los últimos años, los Vehículos Aéreos No Tripulados (VANTs) han adquirido diversas aplicaciones en la ingeniería civil, entre ellas la inspección de infraestructuras. El objetivo del presente artículo es comparar los ortomosaicos obtenidos en los programas Pix4Dmapper y Agisoft Photoscan, considerando los aspectos técnicos y el error en la detección de defectos en pavimento flexible. La metodología se desarrolló en tres etapas: selección de un tramo vial con deterioro, identificando seis defectos; toma de datos en campo y de forma digital (VANT), para este último se consideró un horario (10 a.m.) y tres alturas $(20,30$ y $40 \mathrm{~m})$, y procesamiento digital de las imágenes en ambos programas. Los resultados muestran que la calidad de los ortomosaicos generados es semejante y, en la detección de errores, se reporta que no existe diferencias estadísticamente significativas entre las medias. Si bien ambos programas proporcionan resultados similares y permiten la detección de defectos en pavimento flexible, el programa Pix4Dmapper es más eficiente en tiempo de procesamiento y tamaño de archivo.
\end{abstract}

Palabras clave: Superposición de Imágenes, Tiempo de Procesamiento, Tamaño de Archivo, Error, ANOVA.

\begin{abstract}
In recent years, Unmanned Aerial Vehicles (UAVs) have acquired various applications in civil engineering, including infrastructure inspection. The objective of this article is to compare the orthomosaics obtained in the Pix4Dmapper and Agisoft Photoscan software, considering the technical aspects and the error in the detection of defects in flexible pavement. The methodology was developed in three stages: selection of a road section with deterioration, identifying six defects; data collection in the field and digitally (UAV), for the latter a schedule (10 a.m.) and three heights $(20,30$ and $40 \mathrm{~m})$ were considered, and digital processing of the images in both software. The results show that the quality of the orthomosaics generated is similar and, in the detection of errors, it is reported that there are no statistically significant differences between the means. While both software provides similar results and allow for flaw detection in flexible pavement, the Pix4Dmapper software is more efficient in processing time and file size.
\end{abstract}

Keywords: Image Overlay, Processing Time, File Size, Error, ANOVA. 


\section{Introducción}

La infraestructura vial debe ser segura y estar en buenas condiciones. La funcionalidad debe garantizarse con las tareas de reparación, mantenimiento y rehabilitación, todas de forma efectiva y oportuna. El deterioro de los pavimentos afecta negativamente a las áreas urbanas, como ser movilidad, seguridad, entre otros. Para evitar perjuicios por el mal estado de los pavimentos, es necesario conocer las condiciones reales, este último mediante procesos de inspección y monitoreo (Prosser-Contreras et al., 2020).

En los últimos años, los vehículos aéreos no tripulados (VANTs), comúnmente conocidos como drones, han presentado diversas aplicaciones en la ingeniería civil; entre ellas, la inspección de tramos viales, apuntando a la reducción, tanto de costos como del esfuerzo laboral-operativo (Assis et al., 2019; Kim et al., 2020). Los VANTs presentan varias ventajas en las tareas de inspección: procesamiento de gran cantidad de datos; optimización de los tiempos, se analizan áreas rápidamente; seguridad, pueden ser operados de forma remota o por medio de un plan de vuelo preprogramado, lo que evita el contacto directo del inspector en campo, entre otros (Pinto, 2016).

Los VANTs son herramientas utilizadas para el monitoreo de infraestructuras, colaborando en la identificación de defectos superficiales y evaluación del estado de conservación de tramos viales (Parente et al., 2017; Kim y Irizarry, 2019; Romero-Chambi et al., 2020). Para un análisis cuantitativo es necesario que la adquisición de datos sea precisa y sin interferencia; no obstante, en ocasiones la presencia de obstáculos (árboles, sombras, etc.), dificulta la obtención de los datos, generando complicaciones en la identificación de defectos (Ragnoli et al., 2018).

Otro aspecto importante es referido a los resultados, los cuales son afectados por las características del VANT y programa utilizado para el procesamiento de las imágenes. En este sentido, se recomienda que los programas cuenten con herramientas que permitan mayor precisión y, por tanto, resultados confiables (Mitsikostas, 2017; Fraser y Congalton, 2018).

Dentro de los programas utilizados en la literatura para el procesamiento digital de imágenes se encuentran: Pix4Dmapper (Tan y Li, 2019; Ferrer-González et al., 2020) y Agisoft PhotoScan (Saad y Tahar, 2019; Roberts et al., 2020), estos requieren de licencias pagadas para su uso, pero existen las versiones de prueba para evaluar sus diferentes funcionalidades (Sánchez et al., 2013). Existen programas de uso libre, entre ellos: Opendronemap, Open MVS o Visual SFM; los cuales requieren combinaciones con otros programas de codificación, además de conocimientos avanzados en el área de la informática para generar resultados de calidad. No obstante, la complejidad de estos programas limita su uso para trabajos de ingeniería con drones (Martorell, 2017).

La elección de los programas para el procesamiento de los datos debe estar más allá de la comparación de los costos. Cada proyecto requiere diferentes características técnicas y calidad de los resultados (Oñate, 2015).

Si bien en la literatura los programas Pix4Dmapper y Agisoft PhotoScan han sido utilizados, no existen recomendaciones sobre el uso de uno sobre otro, ni un reporte comparativo de las potencialidades y los resultados obtenidos en la inspección de pavimentos flexibles, específicamente. El objetivo principal del presente artículo es realizar un análisis comparativo entre los programas Agisoft PhotoScan y Pix4Dmapper, considerando aspectos técnicos en la generación del ortomosaico y la identificación de fallas en pavimento flexible. Para la adquisición de datos se tomó en cuenta un horario de vuelo (10:00 am) y tres alturas de vuelo $(20,30,40 \mathrm{~m})$.

\section{Metodología}

Para atender el objetivo de la presente investigación se plantearon tres etapas. La primera consistió en la identificación de un tramo vial de pavimento flexible con señales de deterioro, identificando seis defectos a ser analizados. En la segunda etapa se realizó la adquisición de datos, tanto de forma digital (VANT) como de forma manual (en campo). Finalmente, en la tercera etapa se realizó el procesamiento de los datos por medio de los programas Pix4Dmapper y Agisoft PhotoScan. Se analizaron los resultados de superposición, tamaño 
y tiempo de procesado de los ortomosaicos generados por ambos programas. Posteriormente, se calculó el error (\%) entre la medición digital y en campo. La Figura 1 resume la metodología utilizada.

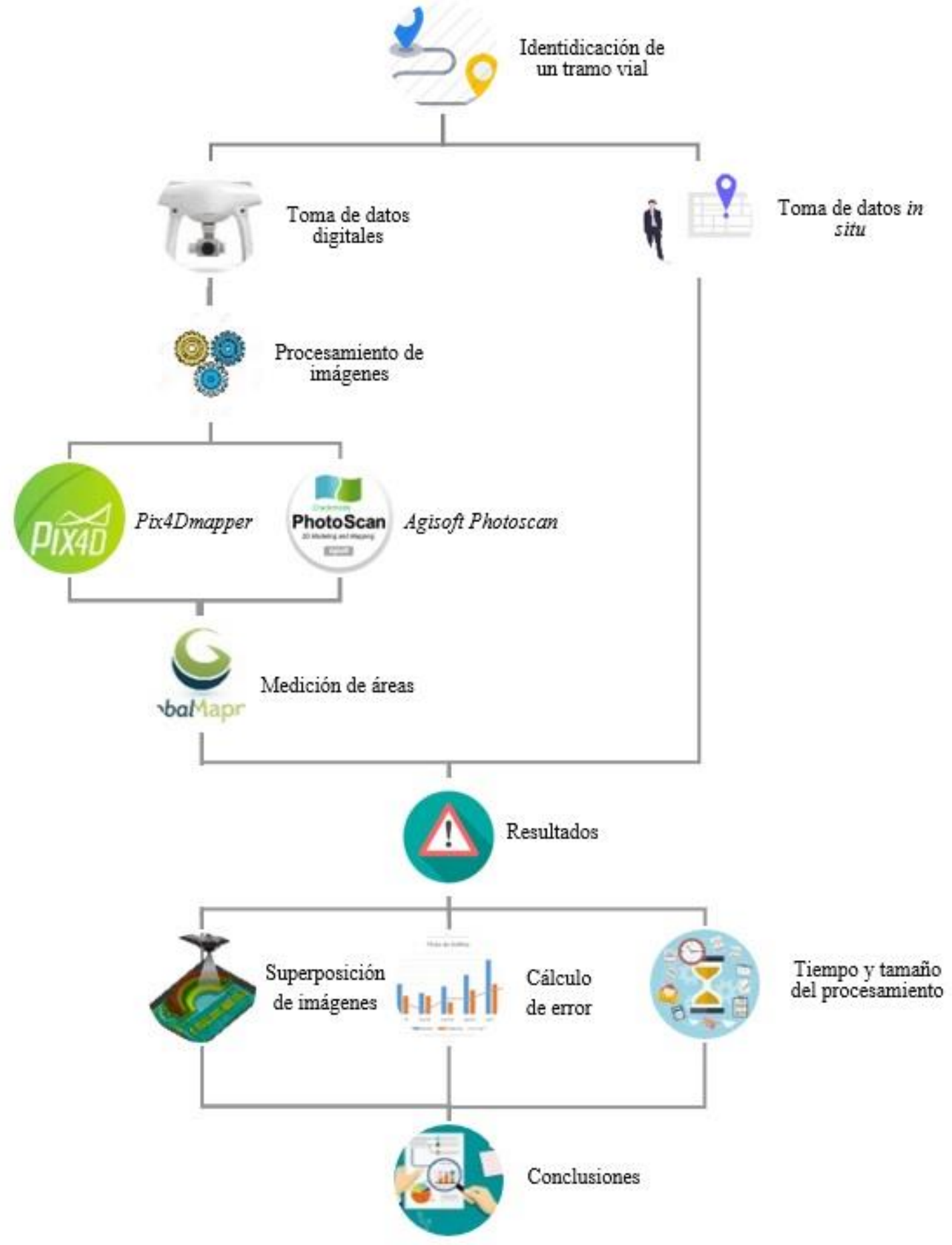

Figura 1 - Metodología utilizada

El tramo seleccionado consta de $100 \mathrm{~m}$ de longitud y se encuentra ubicado en el $\mathrm{km} 3$ de la Avenida Independencia de la ciudad de Cochabamba, Bolivia. El área en estudio se caracteriza por presentar varios defectos sobre la superficie asfáltica, entre ellas: bacheo, piel de cocodrilo y ahuellamiento. Bridle y Porter (2002) señalan que, entre las causas más comunes para el deterioro en pavimento, se encuentran: expansión térmica, lluvia (mal drenaje), uso inadecuado, mayor tráfico que el proyectado, falta de inspección y mantenimiento, entre otras. La Figura 2 presenta la localización del tramo estudiado y los seis defectos identificados. 


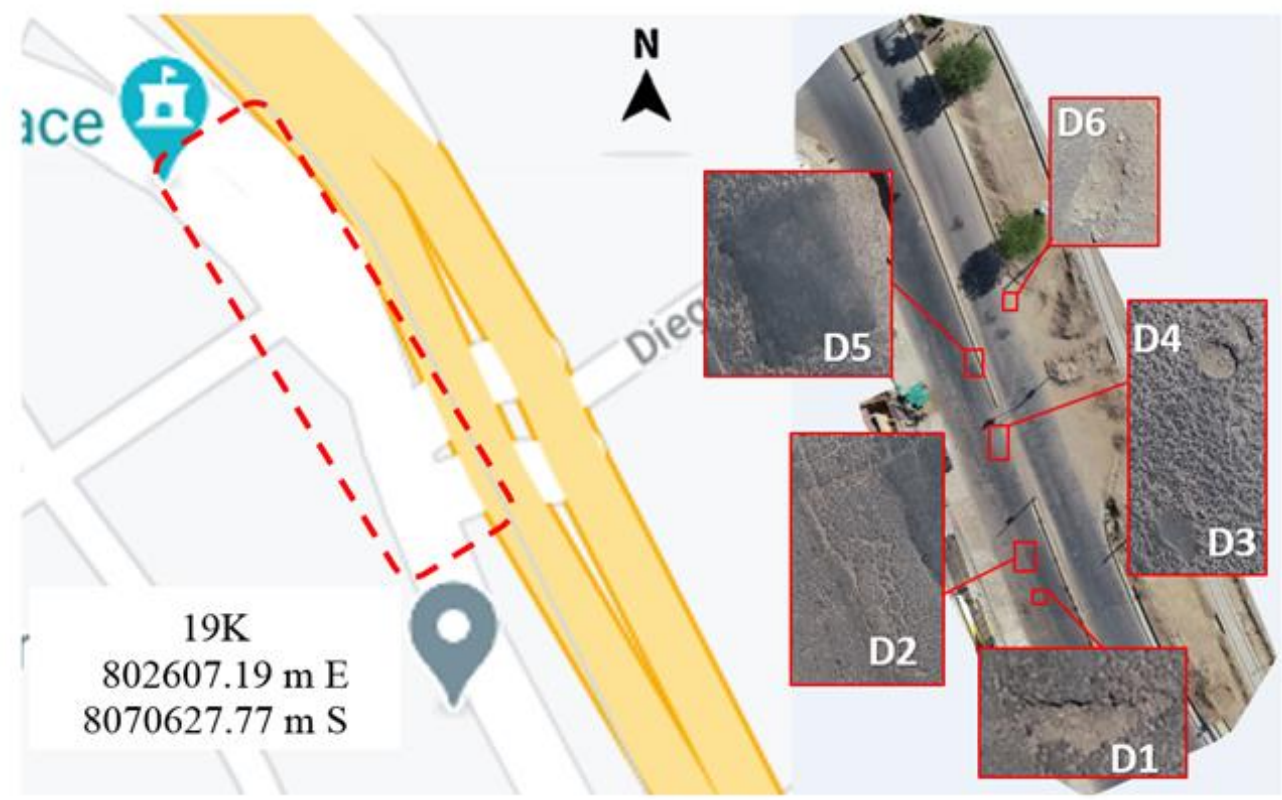

Figura 2 - Localización del área seleccionada y los defectos estudiados

Para la toma de datos se utilizó un VANT de alas rotativas, Phantom 4 Pro de la marca DJI. En la literatura este equipo reporta buenos resultados y confiabilidad (Irizarry y Costa, 2016; Seo et al., 2018; Tan y Li, 2019; Burdziakowski, 2020) y, en comparación con otros VANTs, tiene un menor costo. Entre las especificaciones establecidas por el fabricante (DJI, 2018) se tienen:

- Tiempo máximo de vuelo de 30 minutos por batería.

- Capacidad de visión del sistema: delantera, trasera y hacia abajo.

- Sensores de detección de obstáculos en 4 direcciones: delantera, trasera, derecha e izquierda.

- Sensores de cámara de 1" CMOS con Píxeles efectivos: 20 M.

- Cámara con una máxima resolución de video (4K - 60P).

- Permite realizar vuelos programados que se controlan por medio de aplicaciones.

Para crear el plan de vuelo se utilizó el programa DroneDeploy, en el cual se puede designar el área, definir la ruta de vuelo y calcular el tiempo para la toma de imágenes. Por otra parte, dentro de las configuraciones, el usuario puede modificar: altura de vuelo, velocidad de vuelo, vuelo 2D/3D, superposición, etc. Es necesario verificar las condiciones del lugar para que el VANT opere de manera automática, tanto en la misión como en el despegue y aterrizaje.

Con respecto a la configuración, se deshabilita la opción Live Map HD y la opción 3D mejorado, de modo que las imágenes captadas lleguen a generar una vista en planta. La superposición entre imágenes fue del 85\%, longitudinal y transversal.

Se consideraron tres alturas de vuelo $(20,30$ y $40 \mathrm{~m})$ en el horario de las 10:00 am. El ortomosaico fue generado por medio de los programas: a) Pix4Dmapper (versión 4.4.12), el cual puede crear modelos y mapas digitales 2D/3D georreferenciados y de alta precisión a partir de imágenes tomadas por VANT. b) Agisoft PhotoScan (versión 1.4.5.7354), este llega a generar datos espaciales en 3D para su uso e implementación en Sistemas de Información Geográfica (SIG) y permite realizar mediciones indirectas de objetos de diferentes escalas.

Para la determinación de las áreas de los defectos identificados, se empleó el programa Global Mapper V18, el cual permite analizar y trabajar sobre los ortomosaicos (.TIFF). Se utilizó la herramienta create area feature, esta consiste en dibujar un polígono sobre el área que se desea determinar (Figura 3). Una vez establecidas las áreas, se realizó la comparativa con la medición efectuada en campo.

Las características del ordenador utilizado fueron: MSI GS65 Stealth Thin 8RF, procesador Intel Core i7 de octava generación, de $2.20 \mathrm{GHz}$ (procesador) y de $16 \mathrm{~GB}$ de memoria RAM. 


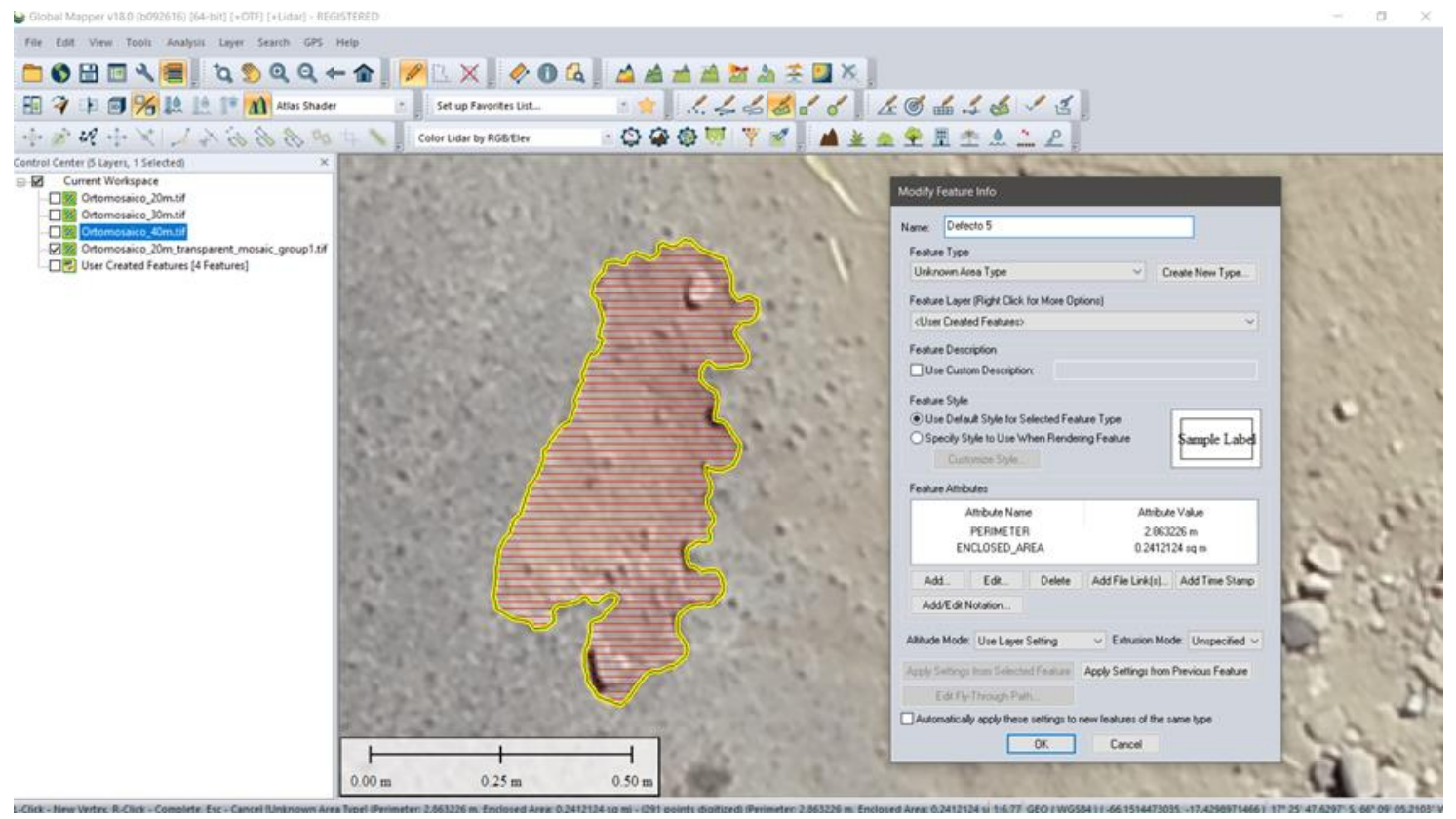

Figura 3 - Medición de áreas con Global Mapper V18

\section{Análisis de los resultados}

\subsection{Superposición de imágenes}

Durante la generación de los ortomosaicos, también se generan reportes de la superposición de las imágenes procesadas, donde se distinguen diferentes calidades a través de colores. En el programa Agisoft PhotoScan se observan tres tonalidades predominantes: azul, verde y naranja que corresponden a traslapes de calidad alta, media y baja, respectivamente. La Figura 4 a muestra el ortomosaico a $20 \mathrm{~m}$ y la Figura $4 \mathrm{~b}$ presenta la superposición de las imágenes, donde predomina la tonalidad azul (alta calidad).
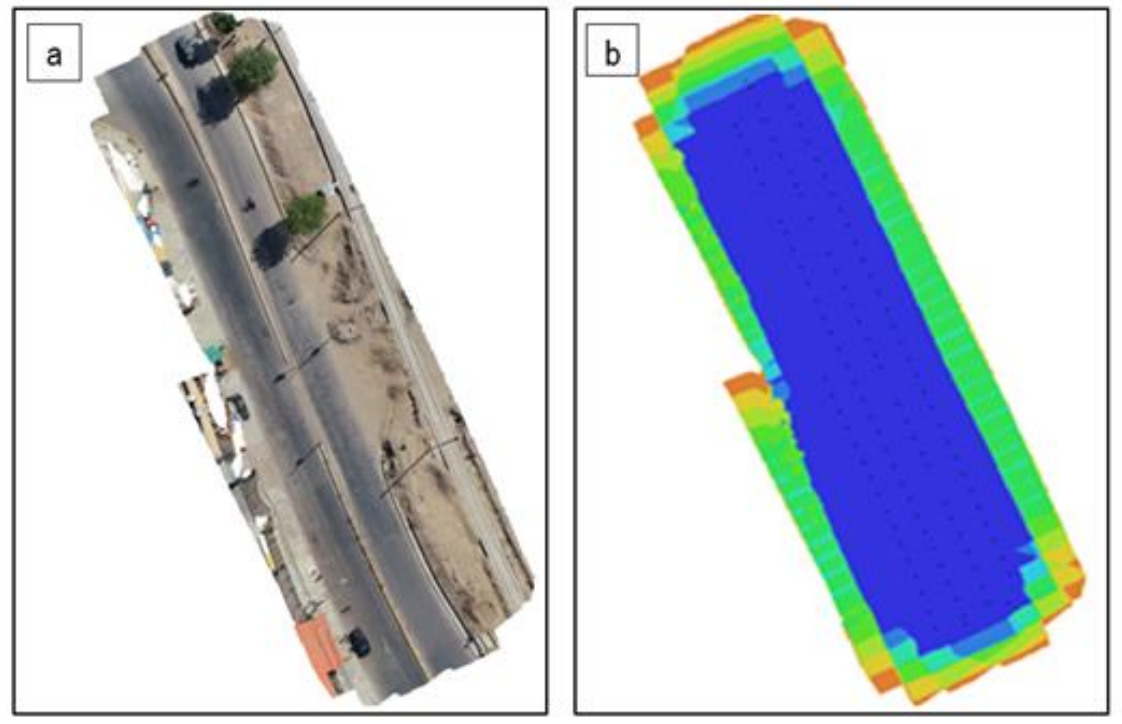

Figura 4 - Resultados de Agisoft PhotoScan para 20 m: a) Ortomosaico y b) Superposición de imágenes

En el caso del programa Pix4Dmapper para la superposición de imágenes, se distinguen los colores verde, amarillo y rojo para alta, media y baja calidad, respectivamente. En la Figura $5 a$ y $5 \mathrm{~b}$ se presentan el ortomosaico y traslape de imágenes a $20 \mathrm{~m}$, con una preponderancia del color verde. En una comparación visual se observa que, Pix4Dmapper, tiene mayor área superpuesta de calidad que Agisoft PhotoScan. 

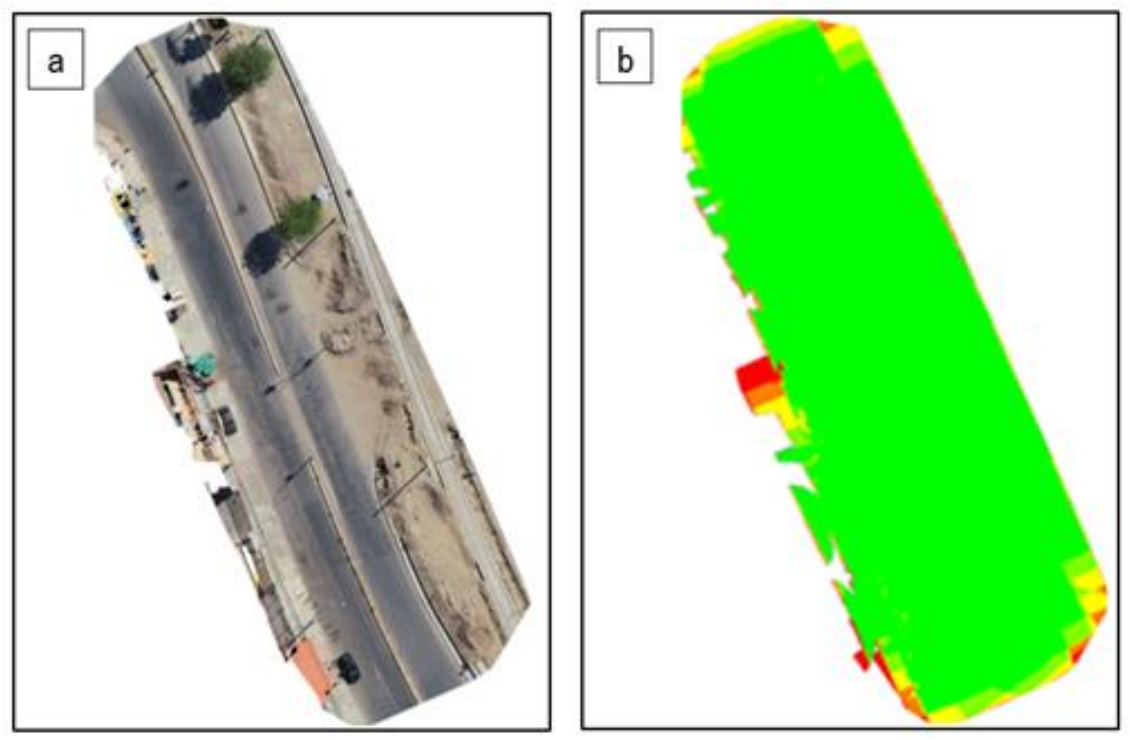

Figura 5 - Resultados de Pix4Dmapper para $20 \mathrm{~m}$ : a) Ortomosaico y b) Superposición de imágenes

Para el ortomosaico a $20 \mathrm{~m}$, el área que genera el programa Pix4Dmapper es $3724.37 \mathrm{~m}^{2}$, siendo $2.22 \%$ menor al área generada por el programa Agisoft PhotoScan, $3808.91 \mathrm{~m}^{2}$. Mientras que, para el área superpuesta de alta calidad, el programa Pix4Dmapper presenta $2990.12 \mathrm{~m}^{2}, 37.06 \%$ por encima del programa Agisoft Photoscan, $2181.57 \mathrm{~m}^{2}$.

El área del ortomosaico generado para las tres alturas de vuelo en Pix4Dmapper, en promedio, es menor en $6.13 \%$ que Agisoft PhotoScan. No obstante, para el promedio del área superpuesta de calidad, el programa Pix4Dmapper supera en $39.27 \%$ al programa Agisoft PhotoScan.

\subsection{Tamaño y tiempo de procesamiento de los ortomosaicos}

El plan de vuelo genera de manera automática el número de imágenes a ser tomadas por el VANT, esta cantidad varía en relación con la altura de vuelo y al área seleccionada. Del Cuadro 1, se logra apreciar que, a menor altura de vuelo, mayor es el número de imágenes y, por tanto, mayor el tiempo de procesamiento para la generación de ortomosaicos. En promedio, los ortomosaicos generados con el programa Pix4Dmapper son menores en tiempo y tamaño en relación con los obtenidos con Agisoft PhotoScan, en $69.10 \%$ y $52.02 \%$, respectivamente.

Cuadro 1 - Comparativa de los ortomosaicos generados

\begin{tabular}{|c|c|c|c|c|}
\hline $\begin{array}{l}\text { Altura de } \\
\text { Vuelo (m) }\end{array}$ & $\begin{array}{l}\text { Número de } \\
\text { imágenes }\end{array}$ & Programa & Tiempo de corrida & $\begin{array}{c}\text { Tamaño de } \\
\text { archivo (MB) }\end{array}$ \\
\hline 20 & \multirow{2}{*}{114} & Pix4dmapper & $01 \mathrm{~h}: 24 \mathrm{~m}: 02 \mathrm{~s}$ & 292 \\
\hline 20 & & Agisoft PhotoScan & $04 \mathrm{~h}: 57 \mathrm{~m}: 13 \mathrm{~s}$ & 572 \\
\hline 30 & \multirow{2}{*}{54} & Pix4dmapper & $00 \mathrm{~h}: 33 \mathrm{~m}: 40 \mathrm{~s}$ & 167 \\
\hline 30 & & Agisoft PhotoScan & $02 \mathrm{~h}: 33 \mathrm{~m}: 20 \mathrm{~s}$ & 346 \\
\hline 40 & \multirow{2}{*}{32} & Pix4dmapper & $00 \mathrm{~h}: 21 \mathrm{~m}: 05 \mathrm{~s}$ & 116 \\
\hline 40 & & Agisoft PhotoScan & $00 \mathrm{~h}: 49 \mathrm{~m}: 15 \mathrm{~s}$ & 260 \\
\hline
\end{tabular}

Para la altura de $40 \mathrm{~m}$, se obtuvo el menor tiempo de procesamiento y tamaño de archivo, ambos con Pix4Dmapper. Mientras que, para la misma altura, Agisoft PhotoScan requirió más tiempo, $133.59 \%$, para la generación del ortomosaico y el tamaño del archivo fue mayor en $124.13 \%$. 
El mayor tiempo de procesamiento y tamaño de archivo fue para la altura de $20 \mathrm{~m}$, ambos obtenidos con Agisoft PhotoScan. Sin embargo, para la misma altura, en Pix4Dmapper, el tiempo de procesamiento y tamaño de ortomosaico fueron menores en $71.72 \%$ y $48.95 \%$, respectivamente.

\subsection{Error en medición de defectos}

La Figura 6 representa los errores (\%) por defecto y altura. Se consideran los seis defectos detectados en el pavimento flexible (bacheo, parcheo y piel de cocodrilo).

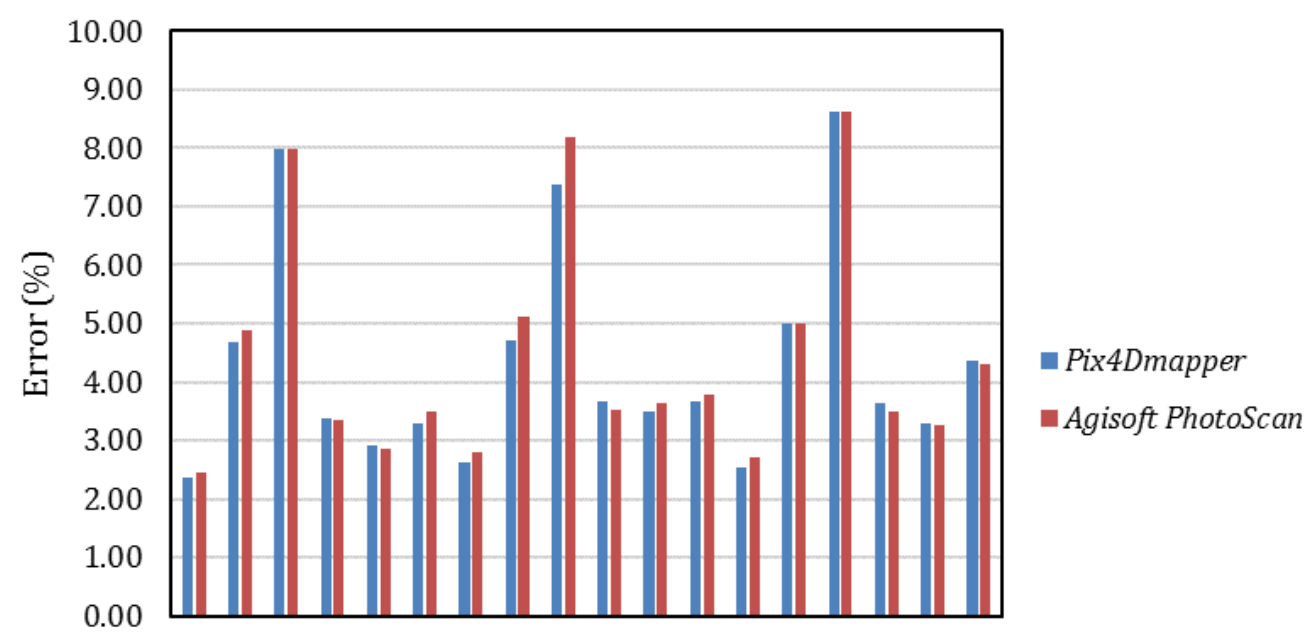

202020202020303030303030404040404040 D1 D2 D3 D4 D5 D6 D1 D2 D3 D4 D5 D6 D1 D2 D3 D4 D5 D6

Altura (m)

Figura 6 - Error por defecto y altura

El error mínimo encontrado fue de $2.36 \%$ (Pix4Dmapper) y 2.45\% (Agisoft PhotoScan), ambos para el D1 a 20 $\mathrm{m}$ de altura. Esta diferencia es mínima, esto se debe a que los ortomosaicos son parecidos en calidad (Figura 7).
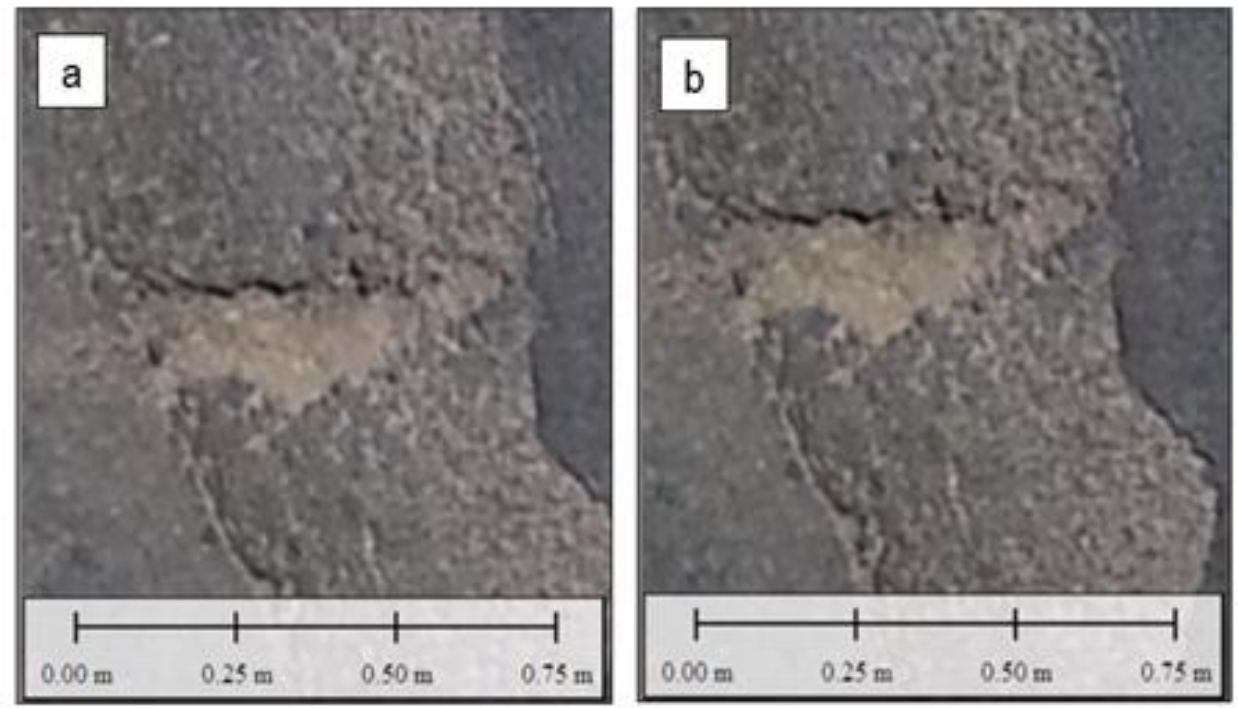

Figura 7 - D1 a 20 m: a) Pix4Dmapper y b) Agisoft PhotoScan 
Por otro lado, en la Figura 8 se presenta el D3 a 40 m de altura, el cual presentó los mayores errores (8.61\%) para los programas utilizados. En este caso (parcheo), ante la presencia constante del color gris y la poca claridad de la imagen (tamaño de píxel), el contorno del defecto no está totalmente definido.
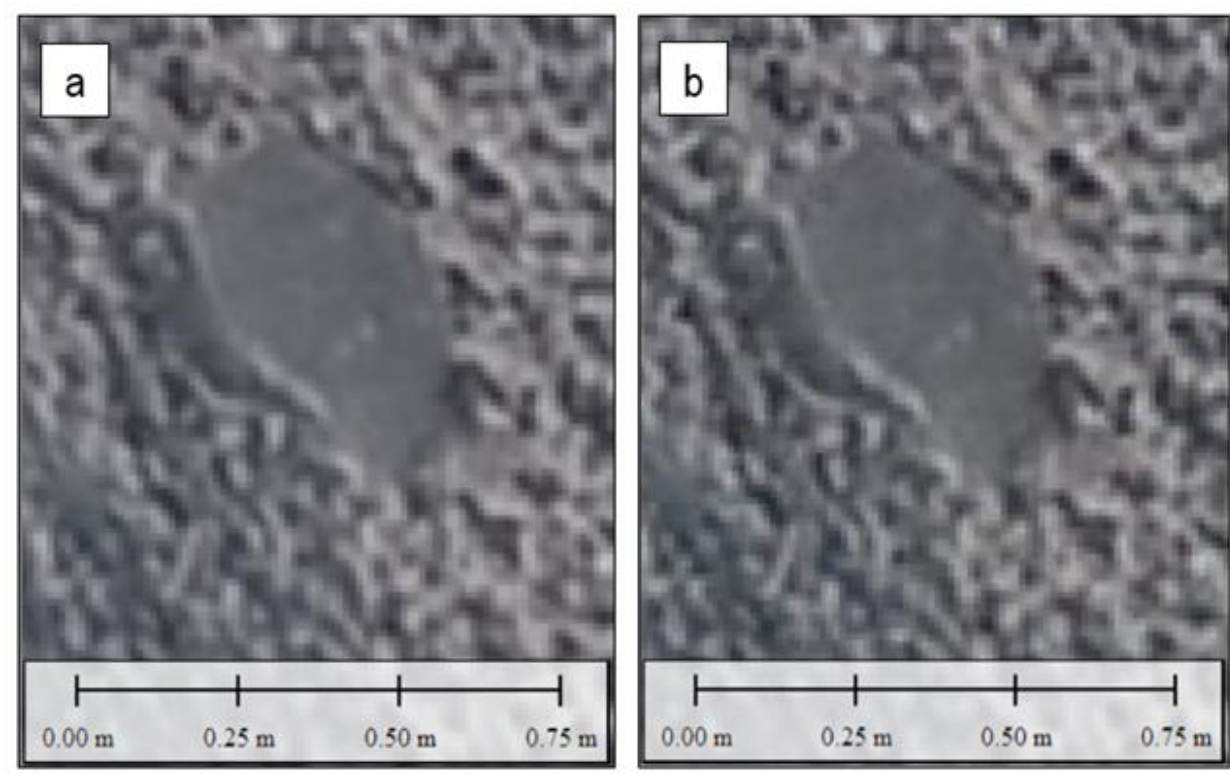

Figura 8 - D3 a 40 m: a) Pix4Dmapper y b) Agisoft PhotoScan

Para comparar si las medias de los errores por ambos programas son iguales o diferentes, se aplicó ANOVA (Analysis of variance). Se plantearon dos hipótesis, nula (H0: las medias son iguales) y alternativa (H1: las medias son diferentes), considerando $\alpha=0.05$. El Cuadro 2 presenta los resultados de ANOVA.

Cuadro 2 - ANOVA para el error por ambos programas

\begin{tabular}{|c|c|c|c|c|c|c|c|c|}
\hline Sources & SS & $\boldsymbol{d f}$ & MS & $\boldsymbol{F}$ & $\begin{array}{c}\boldsymbol{P} \\
\text { value }\end{array}$ & $\boldsymbol{F}$ crit & RMSSE & $\begin{array}{c}\text { Omega } \\
\text { Sq }\end{array}$ \\
\hline Between Groups & 0.0863 & 1 & 0.0863 & 0.0151 & 0.9025 & 4.0981 & 0.0275 & -0.0252 \\
\hline Within Groups & 216.2466 & 38 & 5.6907 & & & & & \\
\hline Total & 216.3330 & 39 & 5.5470 & & & & & \\
\hline
\end{tabular}

Se observa que el valor $F$, cociente entre las varianzas, es 0.015 , indicando que los datos no están dispersos respecto a la media. Luego, $\alpha<P$ value y $F$ crit $>F$, por tanto, se acepta la hipótesis nula (HO), lo que indica que no existen diferencias estadísticamente significativas entre las medias de error de los programas Pix4Dmapper y Agisoft PhotoScan.

\section{Conclusiones}

En la presente investigación se realizó un análisis comparativo entre dos programas comerciales para la generación de ortomosaicos. Se consideraron los aspectos técnicos de los productos y los errores en la detección de defectos en pavimento flexible.

Agisoft PhotoScan genera ortomosaicos con mayor área, pero Pix4Dmapper presenta superposición de alta calidad en mayor porcentaje; no obstante, las áreas con diferencia de traslape se ubican en los márgenes de los ortomosaicos que, en este estudio, no afecta en la detección y medición de defectos en pavimento flexible. 
La resolución de los ortomosaicos está relacionada con la altura de vuelo. A mayor altura de vuelo, la resolución y el número de imágenes van disminuyendo, lo que influye en la detección de defectos, generando mayores errores.

Los ortomosaicos generados por los programas Pix4Dmapper y Agisoft PhotoScan, no presentan diferencias significativas en relación con la calidad visual; sin embargo, Pix4Dmapper es más eficiente en cuestión de tiempo y tamaño de archivo. Con respecto al cálculo del error en la medición de defectos, digital y en campo, se observa que no existen diferencias estadísticamente significativas entre los resultados; en este sentido, cualquiera de los programas analizados serviría para este fin.

Se presentaron resultados acerca del rendimiento de cada programa, considerando aspectos puntuales para la inspección de pavimento flexible. Si bien Pix4Dmapper y Agisoft PhotoScan presentan resultados similares, Pix4Dmapper ofrece mayores ventajas para el análisis de las imágenes obtenidas por VANTs, específicamente, para la detección de defectos en pavimento flexible. No obstante, otros estudios pueden comparar resultados en otras condiciones o características del trabajo a realizar, esto para conocer las potencialidades de cada programa.

\section{Referencias Bibliográficas}

Assis, A., Andrade, C.D., Valente, G.C., Debiasi, P. (2019). Uso de Aeronave Remotamente Pilotada (arp) na identificação de patologias referentes ao pavimento flexível da UFRRJ. Simpósio Brasileiro de Sensoriamento Remoto - SBSR 2019, São José dos Campos, Brasil, 2019.

Bridle, R., Porter, J. (2002). The Motorway Achievement: Frontiers of Knowledge and Practice. United Kingdom: Thomas Telford.

Burdziakowski, P. (2020). Increasing the Geometrical and Interpretation Quality of Unmanned Aerial Vehicle Photogrammetry Products using Super-Resolution Algorithms. Remote Sensing, 12(5), 810.

DJI (2018). Phantom 4 Pro/Prot Manual del usuario V1.2. Disponible en: https://dl.djicdn.com/downloads/phantom_4_pro/20170719/Phantom_4_Pro_Pro_Plus_User_Manual_ ES.pdf/

Ferrer-González, E., Agüera-Vega, F., Carvajal-Ramírez, F., Martínez-Carricondo, P. (2020). UAV Photogrammetry Accuracy Assessment for Corridor Mapping Based on the Number and Distribution of Ground Control Points. Remote Sensing, 12 (15), 2447.

Fraser, B. T., Congalton, R.G. (2018). Issues in Unmanned Aerial Systems (UAS) data collection of complex forest environments. Remote Sensing, 10(6), 908.

Irizarry, J., Costa, D.B. (2016). Exploratory study of potential applications of unmanned aerial systems for construction management tasks. Journal of Management in Engineering, 32(3), 05016001.

Kim, S., Irizarry, J. (2019). Human performance in UAS operations in construction and infrastructure environments. Journal of Management in Engineering, 35(6), 04019026.

Kim, S., Irizarry, J., Kanfer, R. (2020). Multilevel goal model for decision-making in UAS visual inspections in construction and infrastructure projects. Journal of Management in Engineering, 36(4), 04020036.

Martorell, A. (2017). Comparativa de programas para fotogrametría. Disponible en: https://geoinnova.org/blog-territorio/comparativa-de-programas-para-fotogrametria/

Mitsikostas, E. (2017). Monitorización y optimización de tierras con drones y fotogrametría aérea para aplicaciones de precisión en agricultura. Tesis de doctorado. Universidad Politécnica de Valencia, España.

Oñate, M. (2015). Tipología de Aeronaves Pilotadas por Control Remoto. En Fundación de la Energía de la Comunidad de Madrid (Ed.), Los Drones y sus Aplicaciones en Ingeniería Civil (pp. 49-57). Madrid, España: Dirección General de Industria, Energía y Minas de la Comunidad de Madrid.

Parente, D.C., Felix, N.C., Picanço, A.P. (2017). Uso de vehículo aéreo no tripulado (VANT) en la identificación de falla superficial en pavimento asfáltico. Revista Alconpat, 7(2), 160-171. 
Pinto, R. (2016). Drones: La tecnología, ventajas y sus posibles aplicaciones. Disponible en: https://www.sonami.cl/v2/wp-content/uploads/2016/03/09.-Drones-La-tecnologia-ventajas-y-susposibles-aplicaciones.pdf

Prosser-Contreras, M., Atencio, E., Muñoz La Rivera, F., Herrera, R.F. (2020). Use of Unmanned Aerial Vehicles (UAVs) and Photogrammetry to Obtain the International Roughness Index (IRI) on Roads. Applied Sciences, 10(24), 8788.

Ragnoli, A., De Blasiis, M., Di Benedetto, A. (2018). Pavement Distress Detection Methods: A Review. Infrastructures, 3(4), 58.

Roberts, R., Inzerillo, L., Di Mino, G. (2020). Exploiting Low-Cost 3D Imagery for the Purposes of Detecting and Analyzing Pavement Distresses. Infrastructures, 5(1), 6.

Romero-Chambi, E., Villarroel-Quezada, S., Atencio, E., Rivera, M.L. (2020). Analysis of optimal flight parameters of unmanned aerial vehicles (UAVs) for detecting potholes in pavements. Applied Sciences, 10(12), 4157.

Saad, A.M., Tahar, K.N. (2019). Identification of rut and pothole by using multirotor unmanned aerial vehicle (UAV). Measurement, 137, 647-654.

Sánchez, M., Domínguez, E., Iturbe, A., Nava, J. (2013). Experiencias, programas de capacitación y transferencia de tecnología para la obtención de fotografías y videos a partir de VANT. Reunión Nacional SELPET, Percepción Remota y Sistemas de Información Espacial, San Luis Potosí, México, 2013.

Seo, J., Duque, L., Wacker, J.P. (2018). Field application of UAS-based bridge inspection. Transportation Research Record, 2672(12), 72-81.

Tan, Y., Li, Y. (2019). UAV photogrammetry-based 3D road distress detection. ISPRS International Journal of Geo-Information, 8 (9), 409. 